\title{
FINITE DIFFERENCE DISCRETIZATIONS OF SOME INITIAL AND BOUNDARY VALUE PROBLEMS WITH INTERFACE
}

\author{
GEORGIOS D. AKRIVIS AND VASSILIOS A. DOUGALIS
}

\begin{abstract}
We analyze the discretization of initial and boundary value problems with a stationary interface in one space dimension for the heat equation, the Schrödinger equation, and the wave equation by finite difference methods. Extending the concept of the elliptic projection, well known from the analysis of Galerkin finite element methods, to our finite difference case, we prove second-order error estimates in space and time in the $l^{2}$ norm.
\end{abstract}

\section{INTRODUCTION}

Let $\rho>0$ and $x^{*} \in(0,1)$. Given $0<T<\infty$, we consider the following initial and boundary value problem for the heat equation in one space dimension with a stationary (straight) interface. We seek a real-valued function $u=u(x, t)$ for $(x, t) \in[0,1] \times[0, T]$ that satisfies

$$
\begin{array}{lc}
u_{t}=\left(a(x) u_{x}\right)_{x}+b(x, t) u & \text { in }\left(\left[0, x^{*}\right) \cup\left(x^{*}, 1\right]\right) \times[0, T], \\
a\left(x^{*}-\right) u_{x}\left(x^{*}-, t\right)=\rho a\left(x^{*}+\right) u_{x}\left(x^{*}+, t\right), & t \in[0, T], \\
u(x, 0)=v^{0}(x), & x \in[0,1], \\
u(0, t)=u(1, t)=0, & t \in[0, T] .
\end{array}
$$

Here, $a(x)$ is a positive function on $[0,1]$, smooth on $\left[0, x^{*}\right]$ and on $\left[x^{*}, 1\right]$, with a possible discontinuity at $x^{*}$, and $b(x, t)$ is nonpositive, smooth on $\left[0, x^{*}\right] \times[0, T]$ and on $\left[x^{*}, 1\right] \times[0, T]$, with a possible discontinuity on $\left\{x^{*}\right\} \times$ $[0, T]$. We assume that the coefficients and the initial value $v^{0}$ are such that the problem (1.1) possesses a unique solution, continuous on $[0,1] \times[0, T]$ and sufficiently smooth for our purposes on $\left[0, x^{*}\right] \times[0, T]$ and on $\left[x^{*}, 1\right] \times[0, T]$.

We shall approximate the solution of $(1.1)$ by an implicit finite difference scheme, second-order accurate in space and time. In space, we partition the intervals $\left[0, x^{*}\right]$, resp. $\left[x^{*}, 1\right]$, into uniform meshes with meshlengths $h_{-}$, resp. $h_{+}$. Specifically, we let $J, m \in \mathbb{N}$ and $h_{-}, h_{+}$be such that $x^{*}=m h_{-}$, $m h_{-}+(J+1-m) h_{+}=1$, and set $x_{j}:=j h_{-}, j=0, \ldots, m,-$ in particular, $x_{m}=x^{*}$-and $x_{j}:=(j-m) h_{+}+x_{m}, j=m+1, \ldots, J+1$. We also let

Received October 11, 1989; revised March 29, 1990.

1980 Mathematics Subject Classification (1985 Revision). Primary 65M10, 65M15.

Work supported by the Institute of Applied and Computational Mathematics of the Research Center of Crete-FORTH. 
$k:=T / N$ and $t^{n}:=n k, n=0, \ldots, N$, for $N \in \mathbb{N}$. If $u$ is the solution of (1.1), denote $u_{j}^{n}:=u\left(x_{j}, t^{n}\right), j=0, \ldots, J+1, n=0, \ldots, N$, and $u^{n}:=$ $\left(u_{0}^{n}, \ldots, u_{J+1}^{n}\right)^{T} \in \mathbb{R}_{0}^{J+2}$, where $\mathbb{R}_{0}^{J+2}:=\left\{v: v=\left(v_{0}, \ldots, v_{J+1}\right)^{T} \in \mathbb{R}^{J+2}\right.$, $\left.v_{0}=v_{J+1}=0\right\}$.

Our finite difference method, given by (3.1) in $\S 3$ below, produces vectors $U^{n}=\left(U_{0}^{n}, \ldots, U_{J+1}^{n}\right)^{T} \in \mathbb{R}_{0}^{J+2}$ for $n=0, \ldots, N$ that approximate $u^{n}$ and is based in space on a standard difference approximation of (1.1) (cf. [13]), which to the left and right of the interface $x_{m}=x^{*}$ reduces to a simple, locally secondorder accurate, centered scheme. Across the interface it becomes a standard simple relation involving the values of $U^{n}$ at the points $x_{m-1}, x_{m}, x_{m+1}$. The time stepping is effected by a Crank-Nicolson procedure. Hence, the scheme requires solving a $J \times J$ tridiagonal linear system of equations at each time step.

In $\S 3$ we analyze the convergence of this method in a weighted $l^{2}$ norm defined, as is natural for this problem, by

$$
\|v\|_{h}:=\left\{h_{-} \sum_{j=1}^{m-1}\left|v_{j}\right|^{2}+\hat{h}\left|v_{m}\right|^{2}+\rho h_{+} \sum_{j=m+1}^{J}\left|v_{j}\right|^{2}\right\}^{1 / 2},
$$

where we henceforth use the notation $\hat{h}:=\frac{1}{2}\left\{h_{-}+\rho h_{+}\right\} \cdot\|\cdot\|_{h}$ is given here in general for complex vectors in $\mathbb{C}_{0}^{J+2}:=\left\{v: v=\left(v_{0}, \ldots, v_{J+1}\right)^{T} \in \mathbb{C}^{J+2}, v_{0}=\right.$ $\left.v_{J+1}=0\right\}$. We shall use complex-valued vectors for approximating the solution of an analogous problem for the Schrödinger equation in $\S 4$. In Theorem 3.1 we prove the optimal-order error estimate

$$
\max _{n}\left\|U^{n}-u^{n}\right\|_{h} \leq c\left(k^{2}+h^{2}\right),
$$

where $h:=\max \left(h_{-}, h_{+}\right)$and $c$ is a constant independent of $k$ and $h$.

The analysis of numerical methods of finite element and finite difference type for time-dependent interface problems, such as (1.1), has often been considered in the literature. For an error analysis of a finite difference semidiscretization that yields optimal-order error estimates in space, cf. Budak [3]. For full finite difference discretizations see, for instance, Isaacson [5], Samarskii and Fryazinov [10], and Samarskii [8, 9]. Isaacson [5] analyzes the Euler and backward Euler schemes by maximum principle methods and obtains optimal-order error estimates under a restriction implying $k=O\left(h^{2}\right)$. For Crank-Nicolson type schemes, Samarskii and Fryazinov in [10] prove an estimate of $O\left(k^{2}+h^{3 / 2}\right)$, while in [8, 9] Samarskii obtains the optimal-order error estimate. (Strictly speaking, the problem considered in [8-10] is the discretization of equations with discontinuous coefficients, possibly on nonuniform meshes. However the techniques of these papers could be ostensibly extended and applied to the problem at hand.)

The main contribution of the present paper is an apparently new technique for obtaining the optimal-order error estimate (1.2) and, in particular, the 
optimal-order term $h^{2}$ instead of $h^{3 / 2}$; the latter bound can be derived in a straightforward manner and is due of course to the interface condition. Our technique depends on considering first an associated two-point boundary value problem whose (complex-valued in general) solution $w$, continuous on $[0,1]$, satisfies

$$
\begin{aligned}
& \left(a(x) w^{\prime}\right)^{\prime}=f(x) \quad \text { in }\left[0, x^{*}\right) \cup\left(x^{*} 1\right], \\
& a\left(x^{*}-\right) w^{\prime}\left(x^{*}-\right)=\rho a\left(x^{*}+\right) w^{\prime}\left(x^{*}+\right), \\
& w(0)=w(1)=0 .
\end{aligned}
$$

In (1.3), $a$ is as in $(1,1)$, and $f$ is taken to be complex-valued in general (for the purposes of $\S 4$ ), smooth on $\left[0, x^{*}\right]$ and on $\left[x^{*}, 1\right]$, with a possible discontinuity at $x^{*} ; w$ will be supposed to be smooth enough for the purposes of its numerical approximation on $\left[0, x^{*}\right]$ and on $\left[x^{*}, 1\right]$. In $\S 2$ we discretize (1.3) by the analog of the scheme used in the space differencing of (1.1) and prove second-order convergence of the resulting discrete approximation to $w$ in the discrete maximum norm and in a discrete $H^{1}$ norm. This result is standard, and our proof uses ideas from Samarskii [11, pp. 78-82]. (See also Gartland [4] for a recent treatment of finite difference methods for two-point boundary value problems with interface.) The apparently new step here is to use these estimates in the proof of the error bound (1.2) in Theorem 3.1, where, motivated by the elliptic projection concept, well known from the error analysis of Galerkin finite element methods (cf. Wheeler [14]), we define a finite difference analog, an elliptic approximation $W$ to the solution $u$ of (1.1), which satisfies the finite difference scheme for a two-point boundary value problem of the form (1.3) with suitable right-hand side depending on $u$. We then compare $U^{n}$ to $W^{n}$, and the proof of (1.2) is concluded in the standard way by the energy method, with analogous estimates to those of the error analysis of Galerkin methods for parabolic problems (cf. Thomée [12]). It should be pointed out that Samarskii in $[8,9]$ had considered comparing the finite-difference approximation of the parabolic problem to the solution of associated discrete stationary problems with suitable right-hand sides, obtained by splitting the truncation error. His approximations, however, differ from the elliptic approximation used here.

In $\S \S 4$ and 5 the elliptic approximation technique is applied to prove estimates of the form (1.2) for finite difference approximations to the solutions of two other time-dependent problems with stationary interface. In $\S 4$ we consider the following initial and boundary value problem for the Schrödinger equation. Using the notation of (1.1), we seek a complex-valued $u=u(x, t)$ for $(x, t) \in$ $[0,1] \times[0, T]$ that satisfies

$$
\begin{array}{ll}
u_{t}=i \alpha u_{x x}+i \beta(x, t) u & \text { in }\left(\left[0, x^{*}\right) \cup\left(x^{*}, 1\right]\right) \times[0, T], \\
u_{x}\left(x^{*}-, t\right)=\rho u_{x}\left(x^{*}+, t\right), & t \in[0, T], \\
u(x, 0)=v^{0}(x), & x \in[0,1], \\
u(0, t)=u(1, t)=0, & t \in[0, T] .
\end{array}
$$


Here, $i$ is the imaginary unit, $\alpha$ is a nonzero real constant, and $\beta(x, t)$ is real-valued, smooth on $\left[0, x^{*}\right] \times[0, T]$ and on $\left[x^{*}, 1\right] \times[0, T]$, with a possible discontinuity on $\left\{x^{*}\right\} \times[0, T]$. We assume that the coefficients and the (complex-valued) initial data $v^{0}$ are such that (1.4) has a unique solution, continuous on $[0,1] \times[0, T]$ and smooth on $\left[0, x^{*}\right] \times[0, T]$ and on $\left[x^{*}, 1\right] \times$ $[0, T]$. (1.4) occurs in underwater acoustics, as a two-layer horizontal interface problem with a horizontal boundary at $x=1$ and a free surface at $x=0$, for the Schrödinger equation viewed as 'parabolic' approximation to the Helmholtz equation. (For simplicity, we have assumed homogeneous Dirichlet boundary conditions at the endpoints; Neumann or mixed, and also nonhomogeneous, boundary conditions can be analyzed as well, with no additional conceptual complications.) McDaniel and Lee (cf., e.g., [6, 7]) have studied implicit, CrankNicolson-type finite difference approximations for this problem and used them in computations. In fact, our main motivation for the present work was to prove an error estimate of the type (1.2) for the McDaniel-Lee difference scheme (cf. [7]) for which numerical experiments clearly indicate second-order rate of convergence in $x$ and $t$. (Actually, our method is slightly different from the one in [7] in that we use a different evaluation of the coefficient $\beta(x, t)$ to render the scheme conservative. However, the proof of optimal-order convergence for the scheme of [7] is very similar to the one for the scheme at hand.) The proof of (1.2) in the present case of the Schrödinger equation follows broadly the lines of the analogous proof for the heat equation and uses energy techniques similar to the ones that we used for Galerkin methods in [1].

Finally, in $\S 5$, we consider the analogous problem for the wave equation. We seek again a real-valued $u=u(x, t)$ for $(x, t) \in[0,1] \times[0, T]$, satisfying

$$
\begin{array}{lc}
u_{t t}=\left(a(x) u_{x}\right)_{x}+b(x, t) u & \text { in }\left(\left[0, x^{*}\right) \cup\left(x^{*}, 1\right]\right) \times[0, T], \\
a\left(x^{*}-\right) u_{x}\left(x^{*}-, t\right)=\rho a\left(x^{*}+\right) u_{x}\left(x^{*}+, t\right), & t \in[0, T], \\
u(x, 0)=v^{0}(x), & x \in[0,1], \\
u_{t}(x, 0)=v^{1}(x), & x \in[0,1], \\
u(0, t)=u(1, t)=0, & t \in[0, T] .
\end{array}
$$

Here the notation and assumptions on $a(x)$ and $b(x, t)$ are exactly the same as in the case of the heat equation (1.1). The (real-valued) initial data $v^{0}, v^{1}$ and the coefficients are again assumed to be such that a unique solution $u$ of (1.5) exists and is continuous on $[0,1] \times[0, T]$ and sufficiently smooth on $\left[0, x^{*}\right] \times$ $[0, T]$ and on $\left[x^{*}, 1\right] \times[0, T]$. We approximate $(1.5)$ by the classical explicit 5-point Courant-Friedrichs-Lewy difference scheme, modified at the interface $x^{*}$ as in the previous sections, and prove again an error estimate of the form (1.2) under the stability condition, of course, that $\left(\sqrt{|a|}_{\infty} k / \min \left(h_{-}, h_{+}\right)\right) \leq r_{0}$ for any fixed $0<r_{0}<1$. (Here, and in the sequel, $|\cdot|_{\infty}$ will denote the $L^{\infty}(0,1)$ norm.) Now the energy estimate is mildly more complicated, owing 
to the presence of the time-dependent coefficient $b(x, t)$; again, we use ideas from analogous proofs for Galerkin methods, here from Bales, Dougalis, and Serbin [2].

It should be pointed out that our technique of comparing the finite difference solution $U^{n}$ to its elliptic approximation $W^{n}$ is a general device whose application is not limited to interface problems only. For example, it can be used to prove optimal-order convergence of finite difference approximations to initial and boundary value problems for nonlinear PDE's; its application there circumvents many tedious computations involving Taylor expansions.

\section{A TWO-POINT BOUNDARY VALUE PROBLEM}

In this section we consider the discretization of problem (1.3) by a standard three-point finite difference scheme modified at the interface node $x^{*}$. We derive second-order accurate error estimates in the discrete maximum norm and in a discrete $H^{1}$ norm. These results will be used later for deriving error estimates for problems (1.1), (1.4), and (1.5).

In the sequel we shall find it convenient to employ difference quotient notation that conforms to the spatial mesh $\left\{x_{j}\right\}, 0 \leq j \leq J+1$, introduced in $\S 1$, and takes into account the interface at $x^{*}=x_{m}$. For a real or complex $J+2$ vector $\left(v_{0}, v_{1}, \ldots, v_{J+1}\right)^{T}$ we define first the forward and backward difference quotients $\partial v_{j}, \bar{\partial} v_{j}$ as

$$
\begin{aligned}
& \partial v_{j}= \begin{cases}\left(v_{j+1}-v_{j}\right) / h_{-} & \text {if } j=0, \ldots, m-1, \\
\left(v_{j+1}-v_{j}\right) / h_{+} & \text {if } j=m, \ldots, J,\end{cases} \\
& \bar{\partial} v_{j}= \begin{cases}\left(v_{j}-v_{j-1}\right) / h_{-} & \text {if } j=1, \ldots, m, \\
\left(v_{j}-v_{j-1}\right) / h_{+} & \text {if } j=m+1, \ldots, J+1 .\end{cases}
\end{aligned}
$$

Consequently, centered second difference quotients that approximate the function $\left(a(x) v^{\prime}(x)\right)^{\prime}$ at $x_{j} \neq x_{m}$ will be denoted by

$$
\bar{\partial}\left(a_{j+1 / 2} \partial v_{j}\right)=\left\{\begin{array}{r}
{\left[a_{j+1 / 2}\left(v_{j+1}-v_{j}\right)-a_{j-1 / 2}\left(v_{j}-v_{j-1}\right)\right] / h_{-}^{2}} \\
\text { if } j=1, \ldots, m-1, \\
{\left[a_{j+1 / 2}\left(v_{j+1}-v_{j}\right)-a_{j-1 / 2}\left(v_{j}-v_{j-1}\right)\right] / h_{+}^{2}} \\
\text { if } j=m+1, \ldots, J,
\end{array}\right.
$$

where we put $a_{j+1 / 2}=a\left(\left(x_{j}+x_{j+1}\right) / 2\right)$ for $j=0, \ldots, J$. At the interface we let

$$
\delta_{\rho}\left(a_{m+1 / 2} \partial v_{m}\right):=\left\{\rho a_{m+1 / 2}\left(\frac{v_{m+1}-v_{m}}{h_{+}}\right)-a_{m-1 / 2}\left(\frac{v_{m}-v_{m-1}}{h_{-}}\right)\right\} / \hat{h} .
$$


Now let $w_{j}=w\left(x_{j}\right), j=0, \ldots, J+1$, where $w(x)$ is the solution of (1.3). We approximate $\left(w_{0}, \ldots, w_{J+1}\right)^{T}$ by $W \in \mathbb{C}_{0}^{J+2}$, defined by the equations

$$
\begin{aligned}
& \bar{\partial}\left(a_{j+1 / 2} \partial W_{j}\right)=f\left(x_{j}\right) \text { if } 1 \leq j \leq J, j \neq m, \\
& \delta_{\rho}\left(a_{m+1 / 2} \partial W_{m}\right)=\hat{f}\left(x^{*}\right),
\end{aligned}
$$

where we put $\hat{f}\left(x^{*}\right):=\left[h_{+} \rho f\left(x^{*}+\right)+h_{-} f\left(x^{*}-\right)\right] / 2 \hat{h}$. The $J \times J$ tridiagonal matrix of the linear system $(2.1)$ is obviously invertible, i.e., $W$ is uniquely defined. Introduce in $\mathbb{C}_{0}^{J+2}$ the sesquilinear form $a_{h}(\cdot, \cdot)$ by

$$
\begin{aligned}
a_{h}(v, u):=-\left[h_{-} \sum_{j=1}^{m-1} \bar{\partial}\left(a_{j+1 / 2} \partial v_{j}\right) \bar{u}_{j}\right. & +\hat{h} \delta_{\rho}\left(a_{m+1 / 2} \partial v_{m}\right) \bar{u}_{m} \\
& \left.+\rho h_{+} \sum_{j=m+1}^{J} \bar{\partial}\left(a_{j+1 / 2} \partial v_{j}\right) \bar{u}_{j}\right],
\end{aligned}
$$

where the bar over $u_{j}$ denotes complex conjugation. It is easily seen that

$$
\begin{aligned}
& \text { (2.2) } \forall v \in \mathbb{C}_{0}^{J+2} \quad a_{h}(v, v)=h_{-} \sum_{j=0}^{m-1} a_{j+1 / 2}\left|\partial v_{j}\right|^{2}+\rho h_{+} \sum_{j=m}^{J} a_{j+1 / 2}\left|\partial v_{j}\right|^{2}, \\
& \text { (2.3) } \forall v \in \mathbb{C}_{0}^{J+2} \quad a_{h}(v, v) \leq \frac{4|a|_{\infty}}{\left[\min \left(h_{-}, h_{+}\right)\right]^{2}}\|v\|_{h}^{2},
\end{aligned}
$$

and that $a_{h}(\cdot, \cdot)$ is an inner product in $\mathbb{C}_{0}^{J+2}$.

Further, letting $\rho^{*}:=\max (1,1 / \rho)$ and $\underline{a}:=\min _{0 \leq x \leq 1} a(x)$, we see that the following discrete Sobolev-type inequality holds:

$$
\forall v \in \mathbb{C}_{0}^{J+2} \max _{j}\left|v_{j}\right|^{2} \leq\left(\rho^{*} / \underline{a}\right) a_{h}(v, v) .
$$

In fact, for $s \leq m$ we have

$$
\begin{aligned}
2\left|v_{s}\right|^{2}= & \left|\sum_{j=0}^{s-1}\left(v_{j+1}-v_{j}\right)\right|^{2}+\left|\sum_{j=s}^{J}\left(v_{j+1}-v_{j}\right)\right|^{2} \\
\leq & s \sum_{j=0}^{s-1}\left|v_{j+1}-v_{j}\right|^{2}+2(m-s) \sum_{j=s}^{m-1}\left|v_{j+1}-v_{j}\right|^{2} \\
& +2(J+1-m) \sum_{j=m}^{J}\left|v_{j+1}-v_{j}\right|^{2} \\
\leq & 2\left(\rho^{*} / \underline{a}\right) a_{h}(v, v) .
\end{aligned}
$$

A similar inequality holds for $s>m$, and the result follows. A trivial consequence of (2.4), that will be used in $\S 5$, is that

$$
\exists c>0 \forall v \in \mathbb{C}_{0}^{J+2} \quad a_{h}(v, v) \geq c\|v\|_{h}^{2} .
$$

Let $e_{j}:=w_{j}-W_{j}, j=0, \ldots, J+1$. Two approximation results for $W$ are given in the following lemma. 
Lemma 2.1. Let the solution $w$ of $(1.3)$ be continuous in $[0,1]$ and smooth in $\left[0, x^{*}\right]$ and in $\left[x^{*}, 1\right]$. Then, there exists a constant $C$, independent of $h$ and $w$, such that

$$
\max _{0 \leq j \leq J+1}\left|e_{j}\right| \leq C h^{2} \sum_{j=1}^{4}\left|w^{(j)}\right|_{\infty}
$$

and

$$
\left[a_{h}(e, e)\right]^{1 / 2} \leq C h^{2} \sum_{j=1}^{4}\left|w^{(j)}\right|_{\infty},
$$

where $\left|w^{(j)}\right|_{\infty}=\max \left\{\sup _{0 \leq x<x^{*}}\left|w^{(j)}(x)\right|_{\infty}, \sup _{x^{*}<x \leq 1}\left|w^{(j)}(x)\right|_{\infty}\right\}$

Proof. Let us first note that (2.6) follows immediately from (2.7) in view of (2.4). Therefore, it remains to show (2.7). Using (2.1), we have for $1 \leq j \leq J$, $j \neq m$,

$$
\bar{\partial}\left(a_{j+1 / 2} \partial e_{j}\right)=\bar{\partial}\left(a_{j+1 / 2} \partial w_{j}\right)-f\left(x_{j}\right) .
$$

Therefore,

$$
\bar{\partial}\left(a_{j+1 / 2} \partial e_{j}\right)= \begin{cases}h_{-}^{2} g_{j} & \text { if } 1 \leq j \leq m-1, \\ h_{+}^{2} g_{j} & \text { if } m+1 \leq j \leq J\end{cases}
$$

and

$$
\hat{h} \delta_{\rho}\left(a_{m+1 / 2} \partial e_{m}\right)=\rho h_{+}^{2} g_{m}-h_{-}^{2} g_{m}^{\prime},
$$

where, by Taylor's theorem,

$$
\max _{j}\left|g_{j}\right|+\left|g_{m}^{\prime}\right| \leq C \sum_{j=1}^{4}\left|w^{(j)}\right|_{\infty} .
$$

Multiplying (2.8i) by $h_{-} \bar{e}_{j}$ if $j \leq m-1$ and by $\rho h_{+} \bar{e}_{j}$ if $j \geq m+1$, and (2.8ii) by $\bar{e}_{m}$, summing from $j=1$ to $j=J$ and using the definition of $a_{h}$, we obtain

$$
a_{h}(e, e)=h_{-}^{3} \sum_{j=1}^{m-1} g_{j} \bar{e}_{j}+\left(\rho h_{+}^{2} g_{m}-h_{-}^{2} g_{m}^{\prime}\right) \bar{e}_{m}+\rho h_{+}^{3} \sum_{j=m+1}^{J} g_{j} \bar{e}_{j} .
$$

We now essentially derive a discrete $H^{-1}$ estimate for the vector $g$ by introducing an auxiliary vector $\eta \in \mathbb{C}_{0}^{J+2}$ by the relations

$$
\begin{aligned}
& \eta_{j+1}-\eta_{j}=h_{-}^{3} g_{j}, \quad j=1, \ldots, m-1, \\
& \rho \eta_{m+1}-\eta_{m}=\rho h_{+}^{2} g_{m}-h_{-}^{2} g_{m}^{\prime}, \\
& \eta_{j+1}-\eta_{j}=h_{+}^{3} g_{j}, \quad j=m+1, \ldots, J .
\end{aligned}
$$


Obviously,

$$
\begin{aligned}
\eta_{s}= & -h_{+}^{3} \sum_{j=s}^{J} g_{j}, \quad s=m+1, \ldots, J, \\
\eta_{s}= & -\left(\rho h_{+}^{2} g_{m}-h_{-}^{2} g_{m}^{\prime}\right)-h_{-}^{3} \sum_{j=s}^{m-1} g_{j} \\
& -\rho h_{+}^{3} \sum_{j=m+1}^{J} g_{j}, \quad s=1, \ldots, m .
\end{aligned}
$$

Therefore,

$$
\max _{j}\left|\eta_{j}\right| \leq C h^{2} \sum_{j=1}^{4}\left|w^{(j)}\right|_{\infty}
$$

Now, using (2.9), (2.10), we have

$$
\begin{aligned}
a_{h}(e, e) & =\sum_{j=1}^{m-1}\left(\eta_{j+1}-\eta_{j}\right) \bar{e}_{j}+\left(\rho \eta_{m+1}-\eta_{m}\right) \bar{e}_{m}+\rho \sum_{j=m+1}^{J}\left(\eta_{j+1}-\eta_{j}\right) \bar{e}_{j} \\
& =-\sum_{j=0}^{m-1} \eta_{j+1}\left(\bar{e}_{j+1}-\bar{e}_{j}\right)-\rho \sum_{j=m}^{J} \eta_{j+1}\left(\bar{e}_{j+1}-\bar{e}_{j}\right) .
\end{aligned}
$$

Therefore, using the Cauchy-Schwarz inequality, (2.12) and (2.2), we obtain

$$
\begin{aligned}
a_{h}(e, e) \leq & \left\{h_{-} \sum_{j=0}^{m-1}\left|\eta_{j}\right|^{2}+\rho h_{+} \sum_{j=m}^{J}\left|\eta_{j+1}\right|^{2}\right\}^{1 / 2} \\
& \times\left\{h_{-} \sum_{j=0}^{m-1}\left|\partial e_{j}\right|^{2}+\rho h_{+} \sum_{j=m}^{J}\left|\partial e_{j}\right|^{2}\right\}^{1 / 2} \\
\leq & C h^{2}\left[a_{h}(e, e)\right]^{1 / 2} \sum_{j=1}^{4}\left|w^{(j)}\right|_{\infty},
\end{aligned}
$$

and (2.7) follows.

We close this section with two remarks.

Remark 1. There is an easier, direct way to prove (2.6), which avoids (2.7). In fact, from (2.4), (2.9) we obtain

$$
\left(\underline{a} / \rho^{*}\right) \max _{j}\left|e_{j}\right|^{2} \leq \max _{j}\left|e_{j}\right|\left\{h_{-}^{3} \sum_{j=1}^{m-1}\left|g_{j}\right|+\left|\rho h_{+}^{2} g_{m}-h_{-}^{2} g_{m}^{\prime}\right|+\rho h_{+}^{3} \sum_{j=m+1}^{J}\left|g_{j}\right|\right\},
$$

and the result follows. We shall use (2.7) only in the case of the wave equation in $\S 5$. 
Remark 2. Define, for functions $u, v$ in $L^{2}(0,1)$, the inner product

$$
(u, v)_{\rho}=\int_{0}^{x^{*}} u \bar{v} d x+\rho \int_{x^{*}}^{1} u \bar{v} d x .
$$

Then the two-point boundary value problem (1.3) has the following weak formulation: seek $w$ in the Sobolev space $\dot{H}^{1}(0,1)$ such that for each $v \in \dot{H}^{1}(0,1)$

$$
-\left(a w^{\prime}, v^{\prime}\right)_{\rho}=(f, v)_{\rho} .
$$

Consider now the finite-dimensional subspace $S_{h}$ of $\dot{H}^{1}(0,1)$, consisting of piecewise linear functions on the mesh $\left\{x_{j}\right\}, 0 \leq j \leq J+1$, continuous on $[0,1]$ and vanishing at the endpoints 0 and 1 . Endowing $S_{h}$ with the usual hat function basis $\left\{\varphi_{j}\right\}, 1 \leq j \leq J$, one obtains a standard finite element approximation $w_{h} \in S_{h}$ to $w$, given by

$$
-\left(a w_{h}^{\prime}, \varphi_{j}^{\prime}\right)_{\rho}=\left(f, \varphi_{j}\right)_{\rho}, \quad 1 \leq j \leq J .
$$

It can be seen now that equations (2.13) reduce exactly to those in (2.1), provided one uses, in each subinterval $\left[x_{i}, x_{i+1}\right]$, the trapezoidal rule to approximate the integrals in the right-hand side of $(2.13)$ and the midpoint rule to approximate those in the left-hand side. The scheme $(2.1)$ is therefore a finite element scheme, modulo quadrature. One could conceivably, therefore, use the techniques of Wheeler [16] to try to derive discrete maximum norm error estimates for (2.1), taking into account, of course, the interface and the use of numerical quadrature.

\section{The heAT EQUATION}

In this section we consider the discretization of problem (1.1) by the CrankNicolson method modified at the interface node $x_{m}=x^{*}$. For $0 \leq n \leq N, 0 \leq$ $j \leq J+1$, we shall approximate $u_{j}^{n}$ by $U_{j}^{n}$, where $U^{n}=\left(U_{0}^{n}, \ldots, U_{j+1}^{n}\right)^{T} \in$ $\mathbb{R}_{0}^{J+2}$, starting with $U_{j}^{0}:=v_{j}^{0}$. We denote $U_{j}^{n+1 / 2}=\left(U_{j}^{n+1}+U_{j}^{n}\right) / 2, t^{n+1 / 2}=$ $t^{n}+(k / 2), b_{j}^{n+1 / 2}=b\left(x_{j}, t^{n+1 / 2}\right)$ and put $\partial_{\tau} U_{j}^{n}=k^{-1}\left(U_{j}^{n+1}-U_{j}^{n}\right)$. Then, for $0 \leq n \leq N-1$, our scheme becomes

$$
\begin{aligned}
& \partial_{\tau} U_{j}^{n}=\bar{\partial}\left(a_{j+1 / 2} \partial U_{j}^{n+1 / 2}\right)+b_{j}^{n+1 / 2} U_{j}^{n+1 / 2}, \quad 1 \leq j \leq J, j \neq m, \\
& \partial_{\tau} U_{m}^{n}=\delta_{\rho}\left(a_{m+1 / 2} \partial U_{m}^{n+1 / 2}\right)+\hat{b}\left(x^{*}, t^{n+1 / 2}\right) U_{m}^{n+1 / 2},
\end{aligned}
$$

where, by analogy to $\hat{f}, \hat{b}\left(x^{*}, t\right):=\left[h_{-} b\left(x^{*}-, t\right)+\rho h_{+} b\left(x^{*}+, t\right)\right] / 2 \hat{h}$.

Stability. Let

$$
\|g\|:=\left\{\int_{0}^{x^{*}}|g(x)|^{2} d x+\rho \int_{x^{*}}^{1}|g(x)|^{2} d x\right\}^{1 / 2}, \quad g \in L^{2}(0,1) .
$$

Multiplying $u_{t}=\left(a u_{x}\right)_{x}+b u$ first by $u$, integrating by parts over $\left[0, x^{*}\right]$, and then multiplying the equation by $\rho u$, integrating by parts over $\left[x^{*}, 1\right]$ and 
using the interface condition, we easily see that $(d / d t)\|u(\cdot, t)\|^{2} \leq 0$, i.e.,

$$
\|u(\cdot, s)\| \leq\|u(\cdot, t)\|, \quad 0 \leq t \leq s \leq T,
$$

and, in particular,

$$
\forall t \in[0, T] \quad\|u(\cdot, t)\| \leq\left\|v^{0}\right\| .
$$

In the next lemma we prove discrete analogues of $(3.2),(3.3)$ for scheme (3.1).

Lemma 3.1. The approximate solution $U^{n}$ of (3.1) satisfies

$$
\left\|U^{n+1}\right\|_{h} \leq\left\|U^{n}\right\|_{h}, \quad n=0, \ldots, N-1,
$$

and, in particular,

$$
\left\|U^{n}\right\|_{h} \leq\left\|U^{0}\right\|_{h}, \quad n=0, \ldots, N .
$$

Proof. Multiplying the first equation in (3.1) by $h_{-} U_{j}^{n+1 / 2}$ if $j \leq m-1$ and by $\rho h_{+} U_{j}^{n+1 / 2}$ if $j \geq m+1$, and the second by $U_{m}^{n+1 / 2}$, summing from $j=1$ to $j=J$, using (2.2) and the sign conditions on $a, b$, we obtain $\left\|U^{n+1}\right\|_{h}^{2}-$ $\left\|U^{n}\right\|_{h}^{2} \leq 0$, and the results follow.

From this lemma it obviously follows that for each $n=0, \ldots, N-1, U^{n+1}$ exists uniquely as the solution of the $J \times J$ tridiagonal linear system represented by (3.1).

Convergence. The main result in this section is given in the following theorem.

Theorem 3.1. Let $u$ satisfy (1.1) and $U^{n}$ satisfy (3.1). Then there exists a constant $C(u)$, independent of $h$ and $k$, such that

$$
\max _{0 \leq n \leq N}\left\|u^{n}-U^{n}\right\|_{h} \leq C(u)\left(k^{2}+h^{2}\right) \text {. }
$$

Proof. For $0 \leq t \leq T$ let $W(t)=\left(W_{0}, \ldots, W_{J+1}\right)^{T} \in \mathbb{R}_{0}^{J+2}$ be the elliptic approximation to $u(\cdot, t)$ defined by

$$
\begin{aligned}
& \bar{\partial}\left(a_{j+1 / 2} \partial W_{j}\right)=(L u)\left(x_{j}, t\right), \quad 1 \leq j \leq J, j \neq m, \\
& \delta_{\rho}\left(a_{m+1 / 2} \partial W_{m}\right)=(\widehat{L u})\left(x^{*}, t\right),
\end{aligned}
$$

where $L u:=\left(a(x) u_{x}\right)_{x}$. With $W^{n}:=W\left(t^{n}\right)$, denote

$$
\zeta^{n}:=u^{n}-W^{n}, \quad \theta^{n}:=W^{n}-U^{n}, \quad n=0, \ldots, N,
$$

so that $u^{n}-U^{n}=\zeta^{n}+\theta^{n}$. According to Lemma 2.1 we have

$$
\max _{0 \leq n \leq N}\left\|\zeta^{n}\right\|_{h} \leq C h^{2}
$$

Hence, it remains to estimate $\left\|\theta^{n}\right\|_{h}$. Using (3.1), (3.6), (1.1), and letting $\theta_{j}^{n+1 / 2}=\left(\theta_{j}^{n+1}+\theta_{j}^{n}\right) / 2$, we obtain for $1 \leq j \leq J, j \neq m$,

$$
\partial_{\tau} \theta_{j}^{n}=\bar{\partial}\left(a_{j+1 / 2} \partial \theta_{j}^{n+1 / 2}\right)+b_{j}^{n+1 / 2} \theta_{j}^{n+1 / 2}+\omega_{j}^{n},
$$


where for $j \neq m, \omega_{j}^{n}=\omega_{1 j}^{n}+\omega_{2 j}^{n}+\omega_{3 j}^{n}+\omega_{4 j}^{n}+\omega_{5 j}^{n}$, and

$$
\begin{aligned}
\omega_{1 j}^{n}: & =\partial_{\tau} W_{j}^{n}-\partial_{\tau} u_{j}^{n}, \\
\omega_{2 j}^{n} & :=\partial_{\tau} u_{j}^{n}-u_{t}\left(x_{j}, t^{n+1 / 2}\right), \\
\omega_{3 j}^{n}: & :=(L u)\left(x_{j}, t^{n+1 / 2}\right)-\frac{1}{2}\left[(L u)\left(x_{j}, t^{n}\right)+(L u)\left(x_{j}, t^{n+1}\right)\right], \\
\omega_{4 j}^{n}: & =-\frac{1}{2} b_{j}^{n+1 / 2}\left[\left(W_{j}^{n+1}+W_{j}^{n}\right)-\left(u_{j}^{n+1}+u_{j}^{n}\right)\right], \\
\omega_{5 j}^{n}: & : b_{j}^{n+1 / 2}\left[\frac{1}{2}\left(u_{j}^{n+1}+u_{j}^{n}\right)-u\left(x_{j}, t^{n+1 / 2}\right)\right] .
\end{aligned}
$$

Now, we have for $i=1,2,3,4,5$,

$$
\max _{j \neq m}\left|\omega_{i j}^{n}\right| \leq C\left(k^{2}+h^{2}\right) \text {. }
$$

In fact, the terms $\omega_{2 j}^{n}, \omega_{3 j}^{n}, \omega_{5 j}^{n}$ are obviously of $O\left(k^{2}+h^{2}\right)$. An estimate of the same form for $\omega_{4 j}^{n}$ follows immediately from (2.6), while the estimate for $\omega_{1 j}^{n}$ results from $(2.6)$ and the commutativity of the elliptic approximation operator and time differentiation.

At the interface, using again (3.1), (3.6), we have

$$
\begin{aligned}
\partial_{\tau} \theta_{m}^{n} & -\delta_{\rho}\left(a_{m+1 / 2} \partial \theta_{m}^{n+1 / 2}\right)-\hat{b}\left(x^{*}, t^{n+1 / 2}\right) \theta_{m}^{n+1 / 2} \\
= & 2 \partial_{\tau} W_{m}^{n}-\left[(\widehat{L u})\left(x^{*}, t^{n}\right)+(\widehat{L u})\left(x^{*}, t^{n+1}\right)\right] \\
& -\hat{b}\left(x^{*}, t^{n+1 / 2}\right)\left(W_{m}^{n+1}+W_{m}^{n}\right),
\end{aligned}
$$

and, as before, we see that

$$
\partial_{\tau} \theta_{m}^{n}=\delta_{\rho}\left(a_{m+1 / 2} \partial \theta_{m}^{n+1 / 2}\right)+\hat{b}\left(x^{*}, t^{n+1 / 2}\right) \theta_{m}^{n+1 / 2}+\left(h_{-} \omega_{m}^{n}+\rho h_{+} \tilde{\omega}_{m}^{n}\right) / \hat{h}
$$

with

$$
\left|\omega_{m}^{n}\right|+\left|\tilde{\omega}_{m}^{n}\right| \leq C\left(k^{2}+h^{2}\right) .
$$

To derive (3.11ii), we write $\omega_{m}^{n}, \tilde{\omega}_{m}^{n}$ as sums of terms, as was done before. These terms are estimated easily, and (3.11ii) follows because only differences in $t$ are involved. From (3.9) and (3.12) we obtain now, as in the stability proof,

$$
\begin{aligned}
(2 k)^{-1}\left(\left\|\theta^{n+1}\right\|_{h}^{2}-\left\|\theta^{n}\right\|_{h}^{2}\right) \leq & h_{-} \sum_{j=1}^{m-1} \omega_{j}^{n} \theta_{j}^{n+1 / 2}+\left(h_{-} \omega_{m}^{n}+\rho h_{+} \tilde{\omega}_{m}^{n}\right) \theta_{m}^{n+1 / 2} \\
& +\rho h_{+} \sum_{j=m+1}^{J} \omega_{j}^{n} \theta_{j}^{n+1 / 2} .
\end{aligned}
$$

Applying the Cauchy-Schwarz inequality to the right-hand side, and using (3.11i) and (3.11ii), we obtain

$$
\left\|\theta^{n+1}\right\|_{h}-\left\|\theta^{n}\right\|_{h} \leq C k\left(k^{2}+h^{2}\right)
$$


and conclude

$$
\max _{n}\left\|\theta^{n}\right\|_{h} \leq C\left(k^{2}+h^{2}\right) \text {. }
$$

The result now follows from (3.8), (3.13), and the proof is complete.

Remark. In analogy to, and using the notation of Remark 2 in the previous section, consider the Galerkin-finite element formulation of (1.1), with respect to the inner product $(\cdot, \cdot)_{\rho}$, coupled with a Crank-Nicolson type time-stepping procedure, given by

$$
\left(\partial_{\tau} U^{n}, \varphi_{j}\right)_{\rho}+\left(a U_{x}^{n+1 / 2}, \varphi_{j}^{\prime}\right)_{\rho}-\left(b\left(\cdot, t^{n+1 / 2}\right) U^{n+1 / 2}, \varphi_{j}\right)_{\rho}=0,
$$

for $0 \leq n \leq N-1,1 \leq j \leq J$, where $U^{n}=U^{n}(x)$ is the fully discrete approximation of $u\left(x, t^{n}\right)$ in $S_{h}, \partial_{\tau} U^{n}=k^{-1}\left(U^{n+1}-U^{n}\right)$, and $U^{n+1 / 2}=$ $\left(U^{n+1}+U^{n}\right) / 2$. Use the trapezoidal rule on each subinterval $\left[x_{j}, x_{j+1}\right]$ to approximate the integrals in the first and third term in (3.14) (which amounts to lumping into the diagonal the associated matrices), and the midpoint rule to approximate the integrals in the second term. It is seen that (3.14) reduces to the scheme (3.1) which, therefore, may be considered in this sense as a fully discrete finite-element type method, provided one defines $U^{0}$ as the interpolant onto $S_{h}$ of $v^{0}(x)$. Modulo interfaces and quadrature errors, therefore, one could conceivably derive discrete maximum norm error estimates for (3.1), using the techniques of Wheeler [15]. Similar remarks can be made in the case of the wave equation of $\S 5$.

\section{The SCHRÖDINGER EQUATION}

This section is devoted to the discretization of problem (1.4) by the CrankNicolson method modified at the interface node $x^{*}$. For $0 \leq n \leq N, 0 \leq j \leq$ $J+1$, we shall approximate $u_{j}^{n}$ by $U_{j}^{n}$, where $U^{n}=\left(U_{0}^{n}, \ldots, U_{J+1}^{n}\right)^{T} \in \mathbb{C}_{0}^{J+2}$, starting with $U_{j}^{0}:=v_{j}^{0}$. As in the previous section, we let

$$
\begin{array}{cc}
U_{j}^{n+1 / 2}=\left(U_{j}^{n+1}+U_{j}^{n}\right) / 2, & t^{n+1 / 2}=t^{n}+(k / 2), \\
\beta_{j}^{n+1 / 2}=\beta\left(x_{j}, t^{n+1 / 2}\right), & \partial_{\tau} U_{j}^{n}=k^{-1}\left(U_{j}^{n+1}-U_{j}^{n}\right) .
\end{array}
$$

Our scheme, for $0 \leq n \leq N-1$, is:

$$
\begin{aligned}
& \partial_{\tau} U_{j}^{n}=i \alpha \bar{\partial}\left(\partial U_{j}^{n+1 / 2}\right)+i \beta_{j}^{n+1 / 2} U_{j}^{n+1 / 2}, \quad 1 \leq j \leq J, j \neq m, \\
& \partial_{\tau} U_{m}^{n}=i \alpha \delta_{\rho}\left(\partial U_{m}^{n+1 / 2}\right)+i \hat{\beta}\left(x^{*}, t^{n+1 / 2}\right) U_{m}^{n+1 / 2}
\end{aligned}
$$

where we recall that $\bar{\partial}\left(\partial v_{j}\right)=\left(v_{j+1}-2 v_{j}+v_{j-1}\right) / h^{2}$ (where $h=h_{-}$if $j \leq m-1$ and $h=h_{+}$if $\left.j \geq m+1\right)$ and

$$
\delta_{\rho}\left(\partial v_{m}\right)=\left[\rho\left(h_{+}\right)^{-1}\left(v_{m+1}-v_{m}\right)-\left(h_{-}\right)^{-1}\left(v_{m}-v_{m-1}\right)\right] / \hat{h} .
$$

Stability. Multiply first $u_{t}=i \alpha u_{x x}+i \beta u$ by $\bar{u}$, integrate over $\left[0, x^{*}\right]$ by parts and take real parts. Then perform the analogous operations on $\left[x^{*}, 1\right]$ after 
multiplying the equation by $\rho \bar{u}$. Add the resulting equations, use the interface condition and obtain $(d / d t)\|u(\cdot, t)\|^{2}=0$, i.e.,

$$
\|u(\cdot, t)\|=\left\|v^{0}\right\|, \quad t \in[0, T] .
$$

A discrete analog of (4.2) is given in the following lemma.

Lemma 4.1. If $U^{n}$ satisfies (4.1), then

$$
\left\|U^{n}\right\|_{h}=\left\|U^{0}\right\|_{h}, \quad n=0, \ldots, N
$$

Proof. Multiplying the first equation in (4.1) by $h_{-} \bar{U}_{j}^{n+1 / 2}$ if $j \leq m-1$ and by $\rho h_{+} \bar{U}_{j}^{n+1 / 2}$ if $j \geq m+1$, and the second by $\bar{U}_{m}^{n+1 / 2}$, summing from $j=1$ to $J$, using (2.2) and taking real parts, we obtain $\left\|U^{n+1}\right\|_{h}=\left\|U^{n}\right\|_{h}$, and the result follows.

Using Lemma 4.1 , it is easily seen that $U^{n}, n=0, \ldots, N$, are uniquely defined as solutions, for each $n$, of the $J \times J$ tridiagonal linear system represented by (4.1).

Convergence. The proof of the following result is similar to the proof of Theorem 3.1 and will be omitted.

Theorem 4.1. Let $u$ be the solution of (1.4), and $U^{n}$ the solution of (4.1). Then, there exists a constant $C(u)$, independent of $k$ and $h$, such that

$$
\max _{0 \leq n \leq N}\left\|u^{n}-U^{n}\right\|_{h} \leq C(u)\left(k^{2}+h^{2}\right)
$$

\section{The wave equation}

In this section we consider the discretization of problem (1.5) by the standard, explicit Courant-Friedrichs-Lewy five-point scheme, modified at the interface node $x_{m}=x^{*}$. Letting $U_{j}^{0}:=v_{j}^{0}$, we construct approximations $U^{n} \in \mathbb{R}_{0}^{J+2}$ to $u^{n}$ as follows: letting $b_{j}^{n}=b\left(x_{j}, t^{n}\right)$, we first compute $U_{j}^{1}$ by the formulas

$$
\begin{aligned}
& U_{j}^{1}:=U_{j}^{0}+k v_{j}^{1}+\frac{k^{2}}{2}\left[\bar{\partial}\left(a_{j+1 / 2} \partial U_{j}^{0}\right)+b_{j}^{0} U_{j}^{0}\right], \quad 1 \leq j \leq J, j \neq m, \\
& U_{m}^{1}:=U_{m}^{0}+k v_{m}^{1}+\frac{k^{2}}{2}\left[\delta_{\rho}\left(a_{m+1 / 2} \partial U_{m}^{0}\right)+\hat{b}\left(x^{*}, 0\right) U_{m}^{0}\right] .
\end{aligned}
$$

Then, for $1 \leq n \leq N-1$, denoting $\delta_{\tau}^{2} U_{j}^{n}=k^{-2}\left(U_{j}^{n+1}-2 U_{j}^{n}+U_{j}^{n-1}\right)$, we let

$$
\begin{aligned}
& \delta_{\tau}^{2} U_{j}^{n}=\bar{\partial}\left(a_{j+1 / 2} \partial U_{j}^{n}\right)+b_{j}^{n} U_{j}^{n}, \quad 1 \leq j \leq J, j \neq m, \\
& \delta_{\tau}^{2} U_{m}^{n}=\delta_{\rho}\left(a_{m+1 / 2} \partial U_{m}^{n}\right)+\hat{b}\left(x^{*}, t^{n}\right) U_{m}^{n} .
\end{aligned}
$$




\section{Convergence.}

Theorem 5.1. Let $u$ be the solution of (1.5) and $U^{n}$ satisfy (5.1), (5.2). For fixed $0<r_{0}<1$ let $k|a|_{\infty}^{1 / 2} / \min \left(h_{-}, h_{+}\right) \leq r_{0}$. Then there exists a constant $C(u)$, independent of $k$ and $h$, such that

$$
\max _{0 \leq n \leq N}\left\|u^{n}-U^{n}\right\|_{h} \leq C(u)\left(k^{2}+h^{2}\right) .
$$

Proof. Let $W^{n} \in \mathbb{R}_{0}^{J+2}$ be the elliptic approximation to $u\left(\cdot, t^{n}\right)$ given by (3.6) and set

$$
\zeta^{n}:=u^{n}-W^{n}, \quad \theta^{n}:=W^{n}-U^{n}, \quad n=0, \ldots, N,
$$

so that $u^{n}-U^{n}=\zeta^{n}+\theta^{n}$. According to Lemma 2.1 we have

$$
\max _{0 \leq n \leq N}\left\|\zeta^{n}\right\|_{h} \leq C h^{2} \text {. }
$$

Hence, it remains to estimate $\left\|\theta^{n}\right\|_{h}$. Using (5.2), (3.6), we obtain for $1 \leq j \leq$ $J, j \neq m, n=1, \ldots, N-1, L u=\left(a u_{x}\right)_{x}$,

$$
\delta_{\tau}^{2} \theta_{j}^{n}-\bar{\partial}\left(a_{j+1 / 2} \partial \theta_{j}^{n}\right)-b_{j}^{n} \theta_{j}^{n}=\delta_{\tau}^{2} W_{j}^{n}-(L u)\left(x_{j}, t^{n}\right)-b_{j}^{n} W_{j}^{n},
$$

i.e.,

$$
\delta_{\tau}^{2} \theta_{j}^{n}=\bar{\partial}\left(a_{j+1 / 2} \partial \theta_{j}^{n}\right)+b_{j}^{n} \theta_{j}^{n}+\omega_{j}^{n}, \quad j \neq m,
$$

where for $j \neq m, \omega_{j}^{n}=\omega_{1 j}^{n}+\omega_{2 j}^{n}+\omega_{3 j}^{n}$ with

$$
\begin{aligned}
& \omega_{1 j}^{n}:=\delta_{\tau}^{2} W_{j}^{n}-\delta_{\tau}^{2} u_{j}^{n}, \\
& \omega_{2 j}^{n}:=\delta_{\tau}^{2} u_{j}^{n}-u_{t t}\left(x_{j}, t^{n}\right), \\
& \omega_{3 j}^{n}:=b_{j}^{n}\left(u_{j}^{n}-W_{j}^{n}\right) .
\end{aligned}
$$

Now, for $i=1,2,3$,

$$
\max _{j \neq m}\left|\omega_{i j}^{n}\right| \leq C\left(k^{2}+h^{2}\right) .
$$

In fact, $\left|\omega_{2 j}^{n}\right|$ is obviously estimated by $C k^{2}$, and a bound of the form $C\left(k^{2}+h^{2}\right)$ for $\left|\omega_{3 j}^{n}\right|$ follows immediately from Lemma 2.1. Finally, to estimate $\left|\omega_{1 j}^{n}\right|$, we use Lemma 2.1 and the fact that

$$
\delta_{\tau}^{2} u_{j}^{n}=k^{-2} \int_{-k}^{k}(k-|\tau|) u_{t t}\left(x_{j}, t^{n}+\tau\right) d \tau .
$$

Further, for $j=m$, using again (5.2), (3.6), we have

$$
\begin{aligned}
\delta_{\tau}^{2} \theta_{m}^{n} & -\delta_{\rho}\left(a_{m+1 / 2} \partial \theta_{m}^{n}\right)-\hat{b}\left(x^{*}, t^{n}\right) \theta_{m}^{n} \\
& =\delta_{\tau}^{2} W_{m}^{n}-u_{t t}\left(x^{*}, t^{n}\right)+\hat{b}\left(x^{*}, t^{n}\right) u_{m}^{n}-\hat{b}\left(x^{*}, t^{n}\right) W_{m}^{n} .
\end{aligned}
$$


As before, we see that

$$
\delta_{\tau}^{2} \theta_{m}^{n}=\delta_{\rho}\left(a_{m+1 / 2} \partial \theta_{m}^{n}\right)+\hat{b}\left(x^{*}, t^{n}\right) \theta_{m}^{n}+\left(h_{-} \omega_{m}^{n}+\rho h_{+} \tilde{\omega}_{m}^{n}\right) / \hat{h}
$$

with

$$
\left|\omega_{m}^{n}\right|+\left|\tilde{\omega}_{m}^{n}\right| \leq C\left(k^{2}+h^{2}\right)
$$

Introducing the bilinear, symmetric, positive semidefinite form $b_{h}^{n}(\cdot, \cdot)$ in $\mathbb{R}_{0}^{J+2}$ by

$$
b_{h}^{n}(v, w):=-h_{-} \sum_{j=1}^{m-1} b_{j}^{n} v_{j} w_{j}-\hat{h} \hat{b}\left(x^{*}, t^{n}\right) v_{m} w_{m}-\rho h_{+} \sum_{j=m+1}^{J} b_{j}^{n} v_{j} w_{j},
$$

multiplying (5.6) by $h_{-}\left(\theta_{j}^{n+1}-\theta_{j}^{n-1}\right)=h_{-}\left[\left(\theta_{j}^{n+1}-\theta_{j}^{n}\right)+\left(\theta_{j}^{n}-\theta_{j}^{n-1}\right)\right]$ if $j \leq m-1$ and by $\rho h_{+}\left(\theta_{j}^{n+1}-\theta_{j}^{n-1}\right)$ if $j \geq m+1$, multiplying (5.9) by $\left(\theta_{m}^{n+1}-\theta_{m}^{n}\right)$, and summing from $j=1$ to $j=J$, we obtain

$$
\begin{aligned}
k^{-2}\left(\left\|\theta^{n+1}-\theta^{n}\right\|_{h}^{2}-\left\|\theta^{n}-\theta^{n-1}\right\|_{h}^{2}\right) & \\
= & {\left[a_{h}\left(\theta^{n}, \theta^{n+1}-\theta^{n-1}\right)+b_{h}^{n}\left(\theta^{n}, \theta^{n+1}-\theta^{n-1}\right)\right] } \\
& +h_{-} \sum_{j=1}^{m-1} \omega_{j}^{n}\left(\theta_{j}^{n+1}-\theta_{j}^{n-1}\right)+\left(h_{-} \omega_{m}^{n}+\rho h_{+} \tilde{\omega}_{m}^{n}\right)\left(\theta_{m}^{n+1}-\theta_{m}^{n-1}\right) \\
& +\rho h_{+} \sum_{j=m+1}^{J} \omega_{j}^{n}\left(\theta_{j}^{n+1}-\theta_{j}^{n-1}\right) .
\end{aligned}
$$

Therefore, applying the Cauchy-Schwarz inequality and using (5.8), we have

$$
\begin{aligned}
\| \theta^{n+1} & -\theta^{n}\left\|_{h}^{2}-\right\| \theta^{n}-\theta^{n-1} \|_{h}^{2} \\
\leq & -k^{2}\left[a_{h}\left(\theta^{n+1}, \theta^{n}\right)-a_{h}\left(\theta^{n}, \theta^{n-1}\right)\right] \\
& -k^{2}\left[b_{h}^{n}\left(\theta^{n+1}, \theta^{n}\right)-b_{h}^{n}\left(\theta^{n}, \theta^{n-1}\right)\right] \\
& +C k^{2}\left(k^{2}+h^{2}\right)\left\|\theta^{n+1}-\theta^{n-1}\right\|_{h} .
\end{aligned}
$$

Summing from $n=1$ to $n=l \leq N-1$ then yields

$$
\begin{aligned}
\| \theta^{l+1}- & \theta^{l} \|_{h}^{2}+k^{2}\left[a_{h}\left(\theta^{l+1}, \theta^{l}\right)+b_{h}^{l+1}\left(\theta^{l+1}, \theta^{l}\right)\right] \\
\leq & \left\|\theta^{1}-\theta^{0}\right\|_{h}^{2}+k^{2}\left[a_{h}\left(\theta^{0}, \theta^{1}\right)+b_{h}^{0}\left(\theta^{0}, \theta^{1}\right)\right] \\
& +C k\left(k^{2}+h^{2}\right) \max _{0 \leq n \leq N-1}\left\|\theta^{n+1}-\theta^{n}\right\|_{h} \\
& +k^{2} \sum_{n=1}^{l}\left[b_{h}^{n+1}\left(\theta^{n+1}, \theta^{n}\right)-b_{h}^{n}\left(\theta^{n+1}, \theta^{n}\right)\right] .
\end{aligned}
$$


Now, using (2.5),

$$
\begin{aligned}
& \sum_{n=1}^{l}\left[b_{h}^{n+1}\left(\theta^{n+1}, \theta^{n}\right)-b_{h}^{n}\left(\theta^{n+1}, \theta^{n}\right)\right] \\
& \quad \leq C k \sum_{n=1}^{l}\left\|\theta^{n+1}\right\|_{h}\left\|\theta^{n}\right\|_{h} \\
& \quad \leq C k \sum_{n=1}^{l}\left(\left\|\theta^{n+1}+\theta^{n}\right\|_{h}^{2}+\left\|\theta^{n+1}-\theta^{n}\right\|_{h}^{2}\right) \\
& \quad \leq C k \sum_{n=1}^{l}\left[a_{h}\left(\theta^{n+1}+\theta^{n}, \theta^{n+1}+\theta^{n}\right)+\left\|\theta^{n+1}-\theta^{n}\right\|_{h}^{2}\right]
\end{aligned}
$$

Further, using (2.3),

$$
\begin{aligned}
\| \theta^{l+1}- & \theta^{l} \|_{h}^{2}+k^{2}\left[a_{h}\left(\theta^{l+1}, \theta^{l}\right)+b_{h}^{l+1}\left(\theta^{l+1}, \theta^{l}\right)\right] \\
= & \left\|\theta^{l+1}-\theta^{l}\right\|_{h}^{2}+\frac{k^{2}}{4}\left[a_{h}\left(\theta^{l+1}+\theta^{l}, \theta^{l+1}+\theta^{l}\right)-a_{h}\left(\theta^{l+1}-\theta^{l}, \theta^{l+1}-\theta^{l}\right)\right] \\
& +\frac{k^{2}}{4}\left[b_{h}^{l+1}\left(\theta^{l+1}+\theta^{l}, \theta^{l+1}+\theta^{l}\right)-b_{h}^{l+1}\left(\theta^{l+1}-\theta^{l}, \theta^{l+1}-\theta^{l}\right)\right] \\
\geq & {\left[1-r_{0}^{2}+\frac{k^{2}}{4} \min _{x, t} b(x, t)\right]\left\|\theta^{l+1}-\theta^{l}\right\|_{h}^{2}+\frac{k^{2}}{4} a_{h}\left(\theta^{l+1}+\theta^{l}, \theta^{l+1}+\theta^{l}\right) . }
\end{aligned}
$$

Therefore, (5.11) yields

$$
\begin{aligned}
{\left[1-r_{0}^{2}\right.} & \left.+\frac{k^{2}}{4} \min _{x, t} b(x, t)\right]\left\|\theta^{l+1}-\theta^{l}\right\|_{h}^{2}+\frac{k^{2}}{4} a_{h}\left(\theta^{l+1}+\theta^{l}, \theta^{l+1}+\theta^{l}\right) \\
\leq & \left\|\theta^{1}-\theta^{0}\right\|_{h}^{2}+k^{2}\left[a_{h}\left(\theta^{0}, \theta^{1}\right)+b_{h}^{0}\left(\theta^{0}, \theta^{1}\right)\right] \\
& +C k\left(k^{2}+h^{2}\right) \max _{0 \leq n \leq N-1}\left\|\theta^{n+1}-\theta^{n}\right\|_{h} \\
& +C k \sum_{n=1}^{l}\left[\left\|\theta^{n+1}-\theta^{n}\right\|_{h}^{2}+\frac{k^{2}}{4} a_{h}\left(\theta^{n+1}+\theta^{n}, \theta^{n+1}+\theta^{n}\right)\right] .
\end{aligned}
$$

For $k$ sufficiently small, we note that $1-r_{0}^{2}+\frac{k^{2}}{4} \min _{x, t} b(x, t)>0$. Then, applying the discrete Gronwall lemma yields, for $n=1, \ldots, N-1$,

$$
\begin{aligned}
\left\|\theta^{n+1}-\theta^{n}\right\|_{h}^{2} \leq C\left\{\left\|\theta^{1}-\theta^{0}\right\|_{h}^{2}+\right. & k^{2}\left|a_{h}\left(\theta^{0}, \theta^{1}\right)+b_{h}^{0}\left(\theta^{0}, \theta^{1}\right)\right| \\
& \left.+k\left(k^{2}+h^{2}\right) \max _{0 \leq l \leq N-1}\left\|\theta^{l+1}-\theta^{l}\right\|_{h}\right\},
\end{aligned}
$$

i.e.,

$$
\begin{aligned}
& \max _{0 \leq n \leq N-1}\left\|\theta^{n+1}-\theta^{n}\right\|_{h}^{2} \\
& \quad \leq C\left\{\left\|\theta^{1}-\theta^{0}\right\|_{h}^{2}+k^{2}\left|a_{h}\left(\theta^{0}, \theta^{1}\right)+b_{h}^{0}\left(\theta^{0}, \theta^{1}\right)\right|+k^{2}\left(k^{2}+h^{2}\right)^{2}\right\} .
\end{aligned}
$$


Using the Cauchy-Schwarz inequality and (2.3), we have

$$
k^{2}\left|a_{h}\left(\theta^{0}, \theta^{1}-\theta^{0}\right)\right| \leq k^{2} a_{h}\left(\theta^{0}, \theta^{0}\right)+r_{0}^{2}\left\|\theta^{1}-\theta^{0}\right\|_{h}^{2} .
$$

Hence, from (5.12),

$$
\begin{aligned}
& \max _{0 \leq n \leq N-1}\left\|\theta^{n+1}-\theta^{n}\right\|_{h}^{2} \\
& \quad \leq C\left\{\left\|\theta^{1}-\theta^{0}\right\|_{h}^{2}+k^{2}\left[a_{h}\left(\theta^{0}, \theta^{0}\right)+\left|b_{h}^{0}\left(\theta^{0}, \theta^{1}\right)\right|\right]+k^{2}\left(k^{2}+h^{2}\right)^{2}\right\} .
\end{aligned}
$$

According to Lemma 2.1 we have

$$
a_{h}\left(\theta^{0}, \theta^{0}\right)+\left\|\theta^{0}\right\|_{h}^{2} \leq C h^{4}
$$

and using the fact that

$$
\theta_{j}^{1}=\left(W_{j}^{1}-u_{j}^{1}\right)+\left(u_{j}^{1}-U_{j}^{1}\right)=\left(W_{j}^{1}-u_{j}^{1}\right)+O\left(k\left(k^{2}+h^{2}\right)\right),
$$

we get

$$
\left\|\theta^{1}\right\|_{h} \leq C h^{2}
$$

Further, since

$$
\theta_{j}^{1}-\theta_{j}^{0}=\left(W_{j}^{1}-W_{j}^{0}\right)+\left(u_{j}^{1}-u_{j}^{0}\right)+O\left(k\left(k^{2}+h^{2}\right)\right),
$$

we obtain

$$
\left\|\theta^{1}-\theta^{0}\right\|_{h} \leq C k\left(k^{2}+h^{2}\right) .
$$

Finally, using (5.14)-(5.16) in (5.13) yields

$$
\max _{0 \leq n \leq N-1}\left\|\theta^{n+1}-\theta^{n}\right\|_{h} \leq C k\left(k^{2}+h^{2}\right)
$$

i.e.,

$$
\left\|\theta^{n+1}\right\|_{h} \leq\left\|\theta^{n}\right\|_{h}+C k\left(k^{2}+h^{2}\right), \quad n=0, \ldots, N-1
$$

Hence,

$$
\max _{0 \leq n \leq N}\left\|\theta^{n}\right\|_{h} \leq C\left(k^{2}+h^{2}\right)
$$

and the proof is complete.

\section{ACKNOWLEDGMENT}

The authors record their thanks to Professor Vidar Thomée, whose valuable suggestions led to many improvements in the original manuscript.

\section{BIBLIOGRAPHY}

1. G. D. Akrivis and V. A. Dougalis, On a class of conservative, highly accurate Galerkin methods for the Schrödinger equation, RAIRO Modél. Math. Anal. Numér. (to appear).

2. L. A. Bales, V. A. Dougalis, and S. M. Serbin, Cosine methods for second-order hyperbolic equations with time-dependent coefficients, Math. Comp. 45 (1985), 65-89. 
3. B. M. Budak, Homogeneous differential-difference schemes of second order accuracy for parabolic and hyperbolic equations with discontinuous coefficients, Soviet Math. Dokl. 3 (1962), 198-202.

4. E. C. Gartland, Jr., Compact high-order finite differences for interface problems in one dimension, IMA J. Numer. Anal. 9 (1989), 243-260.

5. E. Isaacson, Error estimates for parabolic equations, Comm. Pure Appl. Math. 24 (1961), 381-389.

6. S. T. McDaniel, Applications of energy methods to finite-difference solutions of the parabolic wave equation, Comput. Math. Appl. 11 (1985), 823-829.

7. S. T. McDaniel and D. Lee, $A$ finite difference treatment of interface conditions for the parabolic wave equation: the horizontal interface, J. Acoust. Soc. Amer. 71 (1982), 855858.

8. A. A. Samarskii, A priori estimates for difference equations, U.S.S.R. Comput. Math. and Math. Phys. 1 (1961), 1138-1167.

9. Homogeneous difference schemes on non-uniform nets for equations of parabolic type, U.S.S.R. Comput. Math. and Math. Phys. 3 (1963), 351-393.

10. A. A. Samarskii and I. V. Fryazinov, On the convergence of difference schemes for a heatconduction equation with discontinuous coefficients, U.S.S.R. Comput. Math. and Math. Phys. 1 (1961), 962-982.

11. A. A. Samarskij, Theorie der Differenzenverfahren, Akad. Verlag., Leipzig, 1984.

12. V. Thomée, Galerkin finite element methods for parabolic problems, Lecture Notes in Math., vol. 1054, Springer-Verlag, Berlin, Heidelberg, New York, Tokyo, 1984.

13. R. S. Varga, Matrix iterative analysis, Prentice-Hall, Englewood Cliffs, N. J., 1962.

14. M. F. Wheeler, A priori $L_{2}$ error estimates for Galerkin approximations to parabolic partial differential equations, SIAM J. Numer. Anal. 10 (1973), 723-759.

15. __, $L_{\infty}$ estimates of optimal orders for Galerkin methods for one-dimensional second order parabolic and hyperbolic equations, SIAM J. Numer. Anal. 10 (1973), 908-913.

16. __ An optimal $L_{\infty}$ error estimate for Galerkin approximations to solutions of two-point boundary value problems, SIAM J. Numer. Anal. 10 (1973), 914-917.

Department of Mathematics, University of Crete, Iraklion, Greece

E-mail address, G. D. Akrivis: akrivis@grearn.bitnet

E-mail address, V. A. Dougalis: dougalis@grearn.bitnet 